\title{
The choice of hand positions on the steering wheel
}

Christian Strümpler, TAKATA AG

This manuscript is not available according to publishing restriction.

Thank you for your understanding. 